\title{
The Incidence of Ophthalmic Problems in the Contact Lens Wearing Population
}

\author{
S. J. HARDMAN LEA, M. A. Z. NEUGEBAUER, R. G. SMITH, S. A. VERNON \\ Nottingham
}

\begin{abstract}
Summary
In a prospective study all contact lens wearers presenting to the ophthalmic casualty department of the University Hospital Nottingham within a six month period were examined to determine the number and pattern of ophthalmic problems occurring in this group. This information was then compared with prescribing information from contact lens dispensers in the city. The incidence of problems associated with contact lens wear within the first six months of starting to use a new contact lens was found to be $0.9 \%$ for soft daily wear lenses and $0.8 \%$ for rigid lenses. Reservations about these figures are discussed. The similarity between the incidence for the two types of lenses appears to contradict previous suggestions that soft lens wearers are more at risk of developing complications. It may be that the early period of lens wear represents a favoured time with respect to soft lens induced disease while hard lens problems are more common at this time. Both giant papillary conjunctivitis and corneal ulceration were seen in significant numbers but neither were associated with one particular lens type.
\end{abstract}

The use of contact lenses for the correction of ametropia continues to increase: while no precise figures exist for the United Kingdom, in the United States of America estimates of lens wear have risen from 10 million in 1980 to 20 million in $1985 .{ }^{1}$ Parallel to this increase, concern has grown about the number and variety of contact lens induced ocular disorders. ${ }^{2,3,4}$ Little information is available on either side of the Atlantic about the incidence of such disorders. For any individual who elects to wear contact lenses the chance of incurring disease from the lenses or their solutions remains unknown. In addition, several recent reports have shown severe ocular infections in patients using soft contact lenses, mainly of the extended wear type. ${ }^{5,6}$ It is therefore also of concern that soft contact lens wearers may be more predisposed to ocular problems than hard lens wearers, but this is not yet proven. Little information is thus available on which to base advice about the selection of a particular type of contact lens. This study was therefore established with close co-operation from all contact lens prescribers in a city of population 750,000 to derive a minimum incidence for ophthalmic problems encountered by patients in the first six months of new lens wear and to sub-divide that incidence according to lens type.

\section{Materials and Methods}

All contact lens wearers presenting to the ophthalmic casualty department in a six month period from February to September 1987 were investigated. (This unit provides a 24 hour service not only to the immediate urban population of about three quarters of a million but also to a surrounding area of an additional 250,000). Precise details of lens wear, wearing time, location of initial lens prescriber, months of lens use, cleaning 
method and demographic data were first recorded. Subsequently all patients were examined by one of four ophthalmic surgeons and all pathology described and recorded. When available the contact lenses were also examined. A diagnosis was then determined and appropriate treatment instigated. If no substantive diagnosis was suggested by the individual pattern of clinical symptoms and signs a note was made of 'cause unknown'.

During this period all lens prescribers in Nottingham were contacted and requested to submit details of the number and type of lenses fitted and dispensed to new wearers in the preceding six months. Forty-six out of a total of 47 complied. In addition, in two of the largest contact lens practices analysis of the records of 500 rigid and 500 soft contact lens wearers was undertaken to estimate the number of lens wearers who may abandon contact lens use within the first six months of prescription of a new lens.

The data derived from these sources was handled in two ways. Firstly, an incidence for contact lens associated problems was derived for each type of contact lens simply by dividing the number of patients presenting with problems to eye casualty who had received a new lens within the preceding six months by the number of patients to whom a new lens had been prescribed within the preceding six months in the area, after correction of this

Table I Demographic data

\begin{tabular}{llr}
\hline Sex & male & 115 \\
& female & 131 \\
Age & $<18$ & 7 \\
& $18-35$ & 170 \\
& $35-52$ & 56 \\
\multirow{2}{*}{ Refraction } & $>52$ & 13 \\
& myopia & 210 \\
& aphakia & 20 \\
& hyperopia & 13 \\
& astigmatism & 3 \\
\hline
\end{tabular}

Table II Contact lenses acquired locally in six month trial period

\begin{tabular}{lrrr}
\hline & \multicolumn{3}{c}{ Lens type } \\
& Rigid & Soft & Total \\
\hline New lens casualties & 7 & 11 & 18 \\
New lenses dispensed & 887 & 1132 & 2019 \\
\hline
\end{tabular}

latter figure by an estimate for those patients who might have given up lens wear within that time. By comparison of the incidence for each lens type a relative risk for soft contact lens wear was calculated and $95 \%$ confidence intervals calculated in the usual manner. ${ }^{7}$

While this approach is appropriate for analysis of risk for the individual, it does not provide an overview of the problem in the contact lens wearing population at large. Therefore, the data was also used for assessing the risk of contact lens wear in an alternative way, by deriving the number of weeks for which contact lenses had been worn successfully by the population in our area to whom lenses had been prescribed in the preceding six months and dividing this into the number of weeks for which those who had experienced problems had worn their lenses before problems had arisen. This provided an estimate of the incidence of contact lens associated problems occurring per week of contact lens wear. Again a relative risk for the incidence of soft lenses compared with hard lens wear was calculated and $95 \%$ confidence intervals established for this figure (this requires a modification of the normal method of confidence interval calculation, as the incidence expressed in this form is that of a survival time and statistical methods for confidence interval analysis of incidence is not applicable). ${ }^{8}$

\section{Results}

Two-hundred-and-forty-six patients wearing contact lenses presented to the department in six months out of 10,000 new cases (in a population of approximately one million). Details of demographic data and refractive error are shown in Table I. Of these 246 only 18 had obtained their lenses in Nottingham in the preceding six months. In the same period the local lens prescribers had dispensed 2019 pairs of contact lenses (Table II). Examintion of the records of 250 new hard contact lens wearers and 250 new soft contact lens wearers revealed that 11 of the rigid lens users and four of the soft lens users abandoned their lens wear within the first six months (Table III).

Table IV shows the figures usèd to generate the incidences for rigid or soft contact lens 
Table III Drop out of contact lens wearers within first six months from records of 500 contact lens wearers.

\begin{tabular}{lcc}
\hline & \multicolumn{2}{c}{ Lens Type } \\
& Rigid & Soft \\
\hline Successful wear & 239 & 246 \\
Failed wear in & 11 & 4 \\
first six months & 250 & 250 \\
\hline Total & & \\
\hline
\end{tabular}

Table IV Numbers of contact lens users encountering problems within first six months of new lens wear compared with numbers of lenses prescribed within the same period (after correction for drop out-see Table III: uncorrected number actually prescribed shown in parentheses) to devise an incidence of problems associated with each lens type.

\begin{tabular}{lcc}
\hline & \multicolumn{2}{c}{ Lens Type } \\
& Rigid & Soft \\
\hline $\begin{array}{l}\text { Uneventful wear } \\
\text { Problem within }\end{array}$ & $843(880)$ & $1103(1132)$ \\
first six months & 7 & 11 \\
\hline Total & 850 & 1114 \\
Incidence of problems & 0.0082 & 0.0098 \\
\hline
\end{tabular}

wear. Note that the numbers of those to whom a new lens had been prescribed has been adjusted according to the estimated dropout rate shown in Table III-this must remain an approximation, but the figures are not large enough to affect the eventual incidence. No patient who had abandoned lens wear appeared amongst our patients in eye casualty.

From these results it is seen that the minimum incidence for opthalmic complications is $0.98 \%$ for soft lenses and $0.82 \%$ for hard lenses within the first six months of starting to wear a new lens. The $95 \%$ confidence interval for the $0.16 \%$ difference in problem incidence between the two lens types is from $-0.67 \%$ to $+1.03 \%$, reflecting the relatively small number of cases involved. The relative risk for the incidence of problems in the two types of lens is therefore $0.98 / 0.82=1.2,95 \%$ confidence intervals being from -2.38 to 19.2 .

Using the second form of data analysis, the number of weeks for which hard lenses were successfully worn in our population who had received a new lens within the prior six months was calculated. This figure then acts as the denominator of person-time index and allows us to calculate an incidence of 0.32 problems per 1000 weeks of hard contact lens wear (Table Va). Similarly Table $\mathrm{Vb}$ derives a figure of 0.38 problems per 1000 weeks of soft contact lens wear. The relative risk for soft lens wear compared with rigid lens use employing these figures is therefore 0.38 / $0.32=1.12$. The $95 \%$ confidence interval for this figure is from 0.71 to -1.77 .

The total number and breakdown of diagnoses for each class of lens are arranged in two groups. Table VI shows those problems ascribed to or directly associated with contact lens use. Table VII lists those diagnoses incidental to lens wear.

With regard to specific disease entities,

Table V To show the incidence of problems occurring in each lens type expressed as the number of problems versus total number of hours for lens wear without complications. This latter figure is derived from the number of lenses worn without problems (after correction for drop out) multiplied by 26 weeks, plus the total number of weeks for which those attending casualty had worn lenses prior to onset of symptoms.

\begin{tabular}{lrr}
\hline & \multicolumn{2}{c}{ Lens Type } \\
& Rigid & Soft \\
\hline Problems encountered & 7 & 11 \\
Weeks of uneventful wear & 22,933 & 28,678 \\
\hline $\begin{array}{l}\text { Problems per 1000 weeks } \\
\text { of lens wear }\end{array}$ & 0.32 & 0.38 \\
\hline
\end{tabular}

Table VI Diagnoses associated with contact lens wear

\begin{tabular}{lrrr}
\hline & Rigid & Soft & Total \\
\hline Conjunctivitis & 17 & 39 & 56 \\
Overwear/occlusion & 17 & 28 & 45 \\
Abrasion & 17 & 14 & 31 \\
Acute lens fluid reaction & 2 & 21 & 23 \\
Corneal ulcer & 5 & 4 & 9 \\
GPC & 3 & 5 & 8 \\
Dry eyes & - & 4 & 4 \\
Misplaced lens & 2 & - & 2 \\
Foreign body on lens & 3 & 3 & 6 \\
Lens damage & 2 & 1 & 3 \\
Corneal warpage & 1 & - & 1 \\
Keratoconus & 1 & - & 1 \\
Handling problems & 2 & - & 2 \\
Cause unknown & 8 & 10 & 18 \\
Failed to attend & & & \\
examination & 7 & 9 & 16 \\
\hline & 87 & 138 & 225 \\
\hline
\end{tabular}


Table VII Diagnoses not resulting from lens wear

\begin{tabular}{lccc}
\hline & Rigid & Soft & Total \\
\hline Iritis & - & 4 & 4 \\
Herpes simplex & 2 & 2 & 4 \\
Faulty refraction & 1 & 1 & 2 \\
Blepharitis & - & 3 & 3 \\
Retinal detachment & 2 & 1 & 3 \\
Herpes Zoster & 1 & 0 & 1 \\
Trichiasis & 0 & 1 & 1 \\
Macular disease & 1 & 0 & 1 \\
Chemical burn & 1 & 1 & 2 \\
\hline & 8 & 13 & 21 \\
\hline
\end{tabular}

Table VIII Diagnoses in first six months of new lens wear

\begin{tabular}{lccc}
\hline & Rigid & Soft & Total \\
\hline Conjunctivitis & 2 & 4 & 6 \\
Overwear/occlusion & 2 & 3 & 5 \\
Abrasion & 1 & 1 & 2 \\
Reaction to solution & - & 1 & 1 \\
Handling difficulty & 2 & -2 & 2 \\
Cause unknown & - & 2 & 2 \\
\hline & 7 & 11 & 18 \\
\hline
\end{tabular}

Table VIII shows those diagnosed for the 18 patients who had recently obtained lenses. Tables IX and X illustrate the case histories of those patients diagnosed as having corneal ulceration and giant papillary conjunctivitis respectively.

\section{Discussion}

The main difficulty in establishing an incidence for contact lens associated disease lies in our ignorance of the number and types of contact lenses in use. Not only are these details not available in the United Kingdom as a whole but they remain unknown for that part of the population whch falls under the care of any one ophthalmic unit. Without this basic information it is possible only to comment on the number of lens problems presenting and to compare this with the known general trend of lens usage. On this descriptive basis an increased number of lens related problems has been identified simultaneously with an increase in the use of hydrophilic lenses especially those worn on an extended wear basis. ${ }^{9,10}$ There is also evidence that soft lenses are more liable to bacterial adhe- sion $^{11,12}$ and penetration ${ }^{13}$ and so increased numbers of infective complications associated with soft lens wear might empirically be expected. However, even those reports of association between soft lenses and severe corneal infection ${ }^{14}$ are limited to description of the large number of soft lens wearers in their series-whether this is merely a reflection of the greater use of soft lenses in their study population is impossible to tell without details of that group.

To circumvent this difficulty, this study used information from all the contact lens prescribers in the city of Nottingham so that the exact number and proportion of new lenses dispensed within the preceding six months was established as a fundamental figure. The number of contact lens induced problems occurring with lenses acquired within six months prior to presentation in the same geographical area was then used to derive a general incidence for contact lens induced complications. (This assumes no sudden change in prescribing habits by the local lens

Table IX Patients with corneal ulceration

\begin{tabular}{lcccc}
\hline Lens & Age & Refraction & $\begin{array}{c}\text { Years } \\
\text { worn }\end{array}$ & $\begin{array}{c}\text { Age } \\
\text { of lens } \\
\text { (months) }\end{array}$ \\
\hline GP & 26 & $\mathrm{~m}$ & 9 & 2 \\
Hard & 43 & $\mathrm{~m}$ & 9 & 60 \\
Hard & 50 & $\mathrm{~m}$ & 27 & 36 \\
Hard & 34 & $\mathrm{~m}$ & 10 & 6 \\
Ex Wr & 24 & $\mathrm{~m}$ & 2 & 9 \\
Soft & 31 & $\mathrm{~m}$ & 0.5 & 7 \\
Hard & 23 & $\mathrm{~m}$ & 7 & 24 \\
Soft & 23 & $\mathrm{~m}$ & 3 & 1 \\
Soft & 24 & $\mathrm{~m}$ & 0.7 & 8 \\
\hline
\end{tabular}

(ex wr = extended wear soft contact lens, $\mathrm{m}=$ myopia)

Table X Cases of giant papillary conjunctivitis

\begin{tabular}{ccccrc}
\hline Age & Sex & Lens & Refraction & $\begin{array}{c}\text { Years } \\
\text { worn }\end{array}$ & $\begin{array}{l}\text { Age lens } \\
\text { (months) }\end{array}$ \\
\hline 22 & F & ex wr & m & 5 & 5 \\
49 & M & hard & m & 9 & 3 \\
24 & F & soft & m & 0.5 & 6 \\
27 & M & hard & m & 8 & 96 \\
16 & M & soft & m & 0.5 & 6 \\
45 & M & hard & m & 8 & 48 \\
26 & M & soft & m & 8 & 8 \\
25 & F & soft & aphakia & 2 & 1 \\
\hline
\end{tabular}

(ex wr = extended wear soft lens, $\mathrm{m}=$ myopia) 
prescribers: clearly any patient at the beginning of the study period might have acquired their lenses just prior to the time over which prescribing records were checked.)

The overall incidence of less than $1 \%$ for lens induced disease in this early period of wear must be interpreted with caution; it surely represents a minimum figure. Firstly, only acute problems would tend to present in our series and thus any developing chronic, low grade condition would not be detected. In addition, a certain number of lens induced problems may have been diagnosed and treated by the initial prescriber. Finally, it seems possible that a number of those lens wearers to whom lenses had been dispensed within our area had incurred problems elsewhere and sought attention at other ophthalmic units.

Very little difference was found between the incidence for hard/gas permeable lenses and soft lenses in our series. (It should be noted that no extended wear lenses featured in the 18 problems seen and that fewer than ten extended wear lenses were prescribed in the area in that time). There are several possible factors contributing to this similar incidence, which at first sight appears to contradict all descriptive evidence in the published literature to date. Firstly, a large number of those difficulties that occur with soft contact lenses only develop over the course of time: thiomersal sensitivity, ${ }^{15}$ protein accumulation, vascularisation, microcystic epitheliopathy ${ }^{16}$ and tendency to lens matrix infection. ${ }^{13}$ Conversely, hard lens wearers may well experience more problems with fitting and handling of the lens in this period and thus be over represented. The details of diagnoses made for the 18 cases in question would seem to support these suggestions (Table VIII). Lastly, it is likely that more assiduous lens care is practised in the initial flush of enthusiasm for a new lens. With time, familiarity induces less scrupulous attention to cleaning and disinfection of lenses and so render soft wearers-whose lenses are known to be more susceptible to microbial contamination-more liable to ocular infection. It is certainly well established that on random testing of contact lens cases and solutions, over $50 \%$ are overtly infected. ${ }^{17,18}$
If these factors are significant and the early wearing period represents an ideal time for soft lens wear the incidence of problems associated with this lens type might be expected to rise with the number of months of lens wear. It is not possible to distinguish this in our study protocol and so remains only speculative. Other studies have used the relative risk of soft lens wear as opposed to rigid lens use to derive an estimate of the overall chance of soft lens induced problems ${ }^{19}$ in casualty patients: by this method the relative risk for complications in soft contact lens wear is approximately double that in rigid lens use. However, a further report from the same hospital, but examining aphakic patients attending regular clinic follow up ${ }^{20}$ did not show any difference in the problems encountered in hard or daily wear soft contact lens wearers. It may be that this patient group, as our patients with their new lenses, are more motivated to and informed about scrupulous lens care than the overall group of cases attending eye casualty.

The precise breakdown of lens related diagnoses in our series was made according to clinical symptoms and signs ${ }^{21}$ and is similar to that found elsewhere..$^{22}$ As in other reports, some difficulty was experienced in distinction between overwear, occlusion and mild nonspecific conjunctivitis, and this limits further interpretation of our figures.

Lastly, our analysis of two major complications usually thought to be associated more with soft lens wear-corneal ulceration and giant papillary conjunctivitis-shows that these diseases may occur in any lens type. In this series the numbers are two small to comment on whether they may be more strongly associated with one particular sort of lens. All cases of corneal ulcer were peripheral and superficial in location and all responded well to topical antibiotic therapy. Indeed it was encouraging that over this period no patient required hospitalisation for treatment. In addition, the proportion of eye casualty work load made up by contact lens wearers does not seem to have increased compared with other studies performed over the past few years. ${ }^{17,22}$ However, it must be emphasised that contact lens wearers now number almost 30 million in Europe and the USA and are still increasing (even as this study was being completed 
advertisements appeared in the local press for cosmetic plano tinted lenses). If only a tiny proportion were to develop significant ocular disease the ophthalmic services would be severely strained.

\section{References}

${ }^{1}$ Schiffrin LG and Rich WJ: The Contact Lens Industry: structure, competition and public policy. Health Technology Case Study 31, US Congress, Office of Technology Assessment 1984.

${ }^{2}$ Kirn TF: As numbers of contact lens wearers increase research seeks to determine risk factors, how best to prevent potential eye infections. J Am Med Assn 1987, 258: 17-18.

${ }^{3}$ Schein O, Hibberd P, Kenyon KR: Contact lens complications: incidental or epidemic? Am J Ophthalmol 1986, 102: 116-17.

${ }^{4}$ Galentine PG, Cohen EJ, Laibson PR, Adams CP, Michaud R, Arentsen JJ: Corneal ulcers associated with contact lens wear. Arch Opthalmol 1984, 102: 891-4.

${ }^{5}$ Stenson S: Soft contact lenses and corneal infection. Arch Ophthalmol 1986, 104: 1287-9.

${ }^{6}$ Krachmer H and Purcell JJ: Bacterial corneal ulcers in cosmetic soft contact lens wearers. Arch ophthalmol 1978, 96: 57-61.

${ }^{7}$ In 'Statistics with confidence', Gardiner MJ and Altman DG: BMJ Publications 1989.

${ }^{8}$ Rothman KJ: Modern epidemiology. Little Brown and Co, Boston 1986.

${ }^{9}$ Ormerod LD and Smith RE: Contact lens associates microbial keratitis. Arch Ophthalmol 1986,104: 79-82.

${ }^{10}$ Cohen EJ, Laibson PR, Arentsen JJ, Clemons CS: Corneal ulcers associated with cosmetic extended wear soft contact lenses. Ophthalmology 1987, 94: 109-13.

${ }^{11}$ Duran JA, Refojo MF, Gipson IK, Kenyon KR: Pseudomonas attachment to new hydrogel contact lenses. Arch Ophthalmol 1987, 105: 106-9.

${ }^{12}$ Slusher MM, Myrvik QN, Lewis JC, Gristina AG: Extended wear lenses, biofilm and bacterial adhesion. Arch Ophthalmol 1987, 105: 110-15.

${ }^{13}$ Tripathi RC and Ruben M: Degenerative changes in a soft hydrophilic contact lens: an ultra-structural study. Ophthalmic Res 1972, 4: 185-92.

${ }^{14}$ Chalupa E, Swarbrick HA, Holden BA, Sjöstrand J: Severe corneal infections associated with contact lens wear. Ophthalmology 1987, 94: 17-22.

${ }^{15}$ Mondino BJ, Solomon SM, Zaidman GW: Allergic and toxic reactions in soft contact lens wearers. Surv Ophthalmol 1982, 26: 337-43.

${ }^{16}$ Morgan JF: Complications associated with contact lens solutions. Ophthalmology 1979, 86: 1107-19.

${ }^{17}$ Barry PJ and Ruben M: Contact lens injuries-an analysis of 217 consecutive patients. Contact Lens $J$ 1980, 10: 6-10.

${ }^{18}$ Donzis PB, Mondino BJ, Weissman BA, Bruckner DA: Microbial contamination of contact lens care systems. Am J Ophthalmol 1987, 104: 325-33.

${ }^{19}$ Franks WA, Adams GGW, Dart JKG, Minassian D: Contact lens related disease in an ophthalmic casualty department and the increased risk of soft lens wear. $\mathrm{Br}$ Med J 1988, 297: 524-5.

${ }^{20}$ Dart JKG: Complications of extended wear hydrogel contact lenses. Contax 1986 April 11-19.

${ }^{21}$ Mathews AM, Dart JKG, Sherwood M: Contact lens hygiene and related disease. $\mathrm{J} \mathrm{Br}$ Contact Lens Assn 1983, 6: 36-9.

${ }^{22}$ Vernon SA: Analysis of all new cases seen in a busy regional centre ophthalmic casualty department during 24 week period. J Roy Soc Med 1983, 76: 279-80. 